\title{
A highly efficient method for isolating urinary exosomes
}

\author{
LIUQING HE ${ }^{1,2}$, DING ZHU ${ }^{1,2}$, JUNPU WANG ${ }^{1,2}$ and XIAOYING WU ${ }^{1,2}$ \\ ${ }^{1}$ Department of Pathology, Xiangya Hospital, Central South University, Changsha, Hunan 410008; ${ }^{2}$ Department of Pathology, \\ School of Basic Medical Science, Central South University, Changsha, Hunan 410013, P.R. China
}

Received April 28, 2018; Accepted September 28, 2018

DOI: $10.3892 /$ ijmm.2018.3944

\begin{abstract}
In the present study, a highly efficient method, referred to as optimized ultrafiltration (OUF), was developed. This method is effective for exosome purification and also facilitates clinical work involving substantial urinary exosome isolation. In the OUF method, $0.22-\mu \mathrm{m}$ filters along with a dialysis membrane with a molecular weight cut-off of $10,000 \mathrm{kDa}$ were introduced, in order to remove extracellular microvesicles that were $>200 \mathrm{~nm}$ and concentrate the supernatant up to 1/50 of the initial volume. The existence, purity and production of the exosomes isolated by OUF and conventional ultracentrifugation (UC) were systematically compared by transmission electron microscopy, western blotting and nanoparticle tracking analysis. In addition, colloidal Coomassie-stained gel and reverse transcription-quantitative polymerase chain reaction were used to investigate the stability and integrity of exosomes isolated by these two protocols. The time required and cost of these two methods in the process of isolating urinary exosomes were also estimated. The results indicated that OUF clearly outperforms UC in quantity, quality and biological stability, and this improved method may have extensive applications in the growing fields of clinical biomarker discovery and exosome research.
\end{abstract}

\section{Introduction}

The concept of exosomes was first proposed by Trams et al (1) in 1981, while soon after, exosomes were identified in a study of reticulocyte differentiation as a consequence of multivesicular endosome fusion with the plasma membrane $(2,3)$. Recent studies have revealed that exosomes are vesicles of 30-100 $\mathrm{nm}$ in size (4), which are secreted by cells into the extracellular space and composed of a lipid bilayer containing several specific proteins and RNAs $(5,6)$. These small intraluminal vesicles fuse with the plasma membrane of target cells when released into the extracellular environment (7). Exosomes can be detected in

Correspondence to: Dr Xiaoying Wu, Department of Pathology, Xiangya Hospital, Central South University, 87 Xiangya Road, Changsha, Hunan 410008, P.R. China

E-mail: xyw2007@scu.edu.cn

Key words: exosomes, urine, isolation, ultracentrifugation, high efficiency all bodily fluids, such as urine (8), seminal fluid (9), blood (10) and amniotic fluids (11). In the transmission of information and regulation of cell signal transduction between different cells, exosomes serve a significant role by selectively delivering biologically active substances to the target cells (12). Exosomes also participate in numerous important physiological and pathological processes, including intercellular communication, cell motility, angiogenesis (13), immune response (14), tumor development and metastasis $(15,16)$. Owing to their bioactive cargo, exosomes may serve as messengers and may offer valuable information for the diagnosis and prognosis of disease (17).

Differing from biopsy, the 'liquid biopsy' of exosomes has various advantages, such as being less invasive, and providing easy handling and sampling (18). As one of the body fluids, urine also contains exosomes. Urinary exosomes are not susceptible to external interference due to their natural lipid bilayers. They also contain a large amount of biological information, which can be communicated to target cells $(19,20)$. Therefore, urinary exosomes can serve as a sensitive biomarker of tumors in diagnosis and screening (21).

A large number of strategies have been applied for the isolation of urinary exosomes. However, the high cost of these methods and the low purity of the obtained exosomes remain important challenges, limiting further development of exosomes (22). Among the available strategies, ultracentrifugation (UC) is the most widely used method, which exhibits a low throughput and impure isolated exosomes due to high-molecular-mass proteins, sample heterogeneity and low stability, given the intensiveness of sequential UC steps (23). Thus, there is an urgent need to establish a simple and rapid method of isolating urinary exosomes with high purity, production and biological activity for further applications in research and clinical practice $(24,25)$. Furthermore, a uniform standard regarding the urine collection time in the isolation of urinary exosomes is currently lacking.

The present study reports an optimized ultrafiltration (OUF) method that can effectively isolate urinary exosomes with high purity and quality in a much more simplified way compared with UC. The results suggest that OUF may be useful for further application in the field of exosome study for liquid biopsy, clinical screening and early disease diagnosis.

\section{Materials and methods}

Ethics statement and sample collection. Our previous research has mainly focused on the study of microRNA (miRNA) and 
urine miRNA in ovarian cancer (26-28). In order to determine the clinical value of urinary exosome miRNA for ovarian cancer and other female-specific conditions, therefore, urine samples were collected from young women in the current study. Urine was collected from Chinese female volunteers, aged between 22 and 27 years, subsequent to obtaining written informed consent. Volunteers were requested to collect samples of morning (first urination), afternoon and night urine of one day during four consecutive days. All subjects were relatively young and healthy, without kidney disease, diabetes or other chronic medical history. Approximately $50 \mathrm{ml}$ urine was collected from each subject at each time, and the urine samples from different individuals were not mixed. Specimens were stored at $-80^{\circ} \mathrm{C}$ immediately. The present study was approved by the Ethics Committee of Central South University (Changsha, China) and conducted in adherence with the Declaration of Helsinki.

Exosome purification methods. Urine samples of approximately $50 \mathrm{ml}$ were collected from each individual in the morning (first urination of the day), afternoon (14:00-18:00) and evening (18:00-22:00). The workflows of the two methods for isolating exosomes from urine samples can be briefly summarized as follows: First, urine samples were centrifuged at 2,000 x g for $30 \mathrm{~min}$ at $4^{\circ} \mathrm{C}$ to remove the cells, cell debris, bacteria and the majority of Tamm-Horsfall protein (THP) (29-33). Next, the remaining macropolymers and THP were removed by further centrifugation for $60 \mathrm{~min}$ at $17,000 \mathrm{x} \mathrm{g}$ and $4^{\circ} \mathrm{C}$. The supernatant was split into two fractions with the same volume, namely supernatant 1 (SN1) and SN2. In the UC method, SN1 was directly ultracentrifuged (Beckman L-80XP 70 Ti; Beckman Coulter, Inc. Brea, CA, USA) at $200,000 \mathrm{x}$ g for $60 \mathrm{~min}$ at $4^{\circ} \mathrm{C}$ in order to collect exosome pellets. In comparison, in the OUF method, SN2 was passed through a $0.22-\mu \mathrm{m}$ filter to remove proteins with diameters of $>0.22 \mu \mathrm{m}$. The filtered solution was then centrifuged at $3,000 \mathrm{x} \mathrm{g}$ for $30 \mathrm{~min}$ at $4^{\circ} \mathrm{C}$ in the dialysis tube with a molecular weight cut-off (MWCO) membrane (Merck KGaA, Darmstadt, Germany). In these two steps, SN2 was passed through two types of filter to excess interference from soluble protein and then concentrated to 1/50 of the original volume. Next, the concentrated supernatant (CSN) was incubated with ExoQuick-TC ${ }^{\text {TM }}$ exosome precipitation solution (cat. no. EXOTC50A-1; System Biosciences, Palo Alto, CA, USA) for $30 \mathrm{~min}$ at $4^{\circ} \mathrm{C}$. Subsequently, the mixture was spun at $15,279 \mathrm{x} \mathrm{g}$ for $2 \mathrm{~min}$ at $4^{\circ} \mathrm{C}$ to harvest the yellow pellets of exosomes (Figs. 1 and $2 \mathrm{~A}$ ).

Nanoparticle tracking analysis (NTA). A NanoSight LM10 (Malvern Panalytical Ltd., Malvern, UK) was used to detect the size of the exosomes. Briefly, $10 \mu 1$ exosomes samples were diluted to 1:100 in 1X PBS. Next, the mixture was placed into the $1 \mathrm{ml}$ injector and injected into the nanoparticle tracking analyzer in order to analyze the size of the exosomes.

Transmission electron microscopy (TEM). Exosome samples were fixed with $1 \%$ glutaraldehyde in PBS at an optimal concentration. The mixture was then spotted onto a 300 mesh carbon/formvar-coated grids and dried at room temperature. Next, the grids were washed with PBS and stained for contrast using uranyl acetate in water for $10 \mathrm{~min}$. Subsequent to staining, samples were imaged by TEM (FEI, Hillsboro, OR,
USA), and images were captured with an AMT CCD Camera (Advanced Microscopy Techniques, Danvers, MA, USA).

Protein assay, colloidal Coomassie-stained gel and western blotting. Exosomes were lysed in radioimmunoprecipitation assay lysis buffer (Thermo Fisher Scientific, Inc., Waltham, MA, USA). Protein concentrations were determined with a bicinchoninic acid protein assay. Denaturation of protein was obtained by appropriately mixing with $5 \mathrm{mM}$ $\beta$-mercaptoethanol (Thermo Fisher Scientific, Inc.) in a water bath at $98^{\circ} \mathrm{C}$ for $10 \mathrm{~min}$. A total of $20 \mu \mathrm{g}$ protein (for each sample) was separated by $8 \%$ SDS-PAGE, and then stained at room temperature for $1 \mathrm{~h}$ in $0.01 \%(\mathrm{w} / \mathrm{v})$ Coomassie Brilliant Blue G-250 (Thermo Fisher Scientific, Inc.), 4.7\% (w/v) ethanol and $8.5 \%(\mathrm{w} / \mathrm{v})$ phosphoric acid as previously described (34), or transferred onto $0.45 \mu \mathrm{m}$ polyvinylidene fluoride membranes (Merck KGaA) by wet electrophoretic transfer. For western blotting, the protein blot was blocked with $5 \%$ skimmed milk for $2 \mathrm{~h}$ at room temperature and incubated overnight at $4^{\circ} \mathrm{C}$ with the following primary antibodies, according to manufacturer's protocol: Anti-CD63 (cat. no. Ab134045; 1:500; Abcam, Cambridge, MA, USA) and anti-heat shock protein 70 (anti-Hsp70; cat. no. Ab2787; 1:500; Abcam). Following washing in $0.1 \%$ PBS-Tween 20 , the protein blot was incubated with horseradish peroxidase-conjugated goat anti-mouse IgG1 secondary antibody (cat. no. SA00001-1; 1:5,000; ProteinTech, Chicago, IL, USA). Subsequently, the protein blot was washed four times in $0.1 \%$ PBS-Tween 20 on an orbital shaker. Enhanced chemiluminescence mixture (Sigma-Aldrich; Merck $\mathrm{KGaA}$ ) was then added to the blot, and images were captured using a gel imaging system (Bio-Rad Laboratories, Inc., Hercules, CA, USA).

RNA isolation and reverse transcription-quantitative polymerase chain reaction ( $R T-q P C R)$ analysis. Total RNA was isolated using TRIzol Plus RNA purification kit (Thermo Fisher Scientific, Inc.). RNA concentration was measured with a NanoDrop ${ }^{\circledR}$ ND-1000 (Thermo Fisher Scientific, Inc.). Subsequently, reverse transcription was performed using All-in-One ${ }^{\mathrm{TM}}$ miRNA First-Strand cDNA Synthesis Kit (cat. no. QP013; GeneCopoeia Biosciences, Guangzhou, China) according to manufacturer's protocol. Next, qPCR was conducted according to the protocol described in the All-in-One ${ }^{\mathrm{TM}}$ miRNA qPCR kit (cat. no. QP016; GeneCopoeia Biosciences) in a $20 \mu \mathrm{l}$ reaction tube. The thermocycling conditions of qPCR were as follows: Initial denaturation at $95^{\circ} \mathrm{C}$ for $10 \mathrm{~min}$; followed by 40 cycles of denaturation at $95^{\circ} \mathrm{C}$ for $10 \mathrm{sec}$, annealing at $60^{\circ} \mathrm{C}$ for $30 \mathrm{sec}$ and extension at $72^{\circ} \mathrm{C}$ for $38 \mathrm{sec}$. The primers of miR-205, miR-7-5p and RNU6 were purchased as ready-to-order specific primer pairs (sequences unavailable) from GeneCopoeia, Inc. (Rockville, MD, USA). Amplification was performed with an ABI-7500 machine (Applied Biosystems; Thermo Fisher Scientific, Inc.). The data of RT-qPCR were analyzed with the SDS relative quantification software, version 2.2.2 (Thermo Fisher Scientific, Inc.). Relative fold-changes in expression were calculated using the $2^{-\Delta \Delta \mathrm{Cq}}$ method (35).

Statistical analysis. Values are presented as the mean \pm standard deviation. Statistical analysis was performed using IBM SPSS software, version 20.0 (IBM Corp., Armonk, NY, USA). 


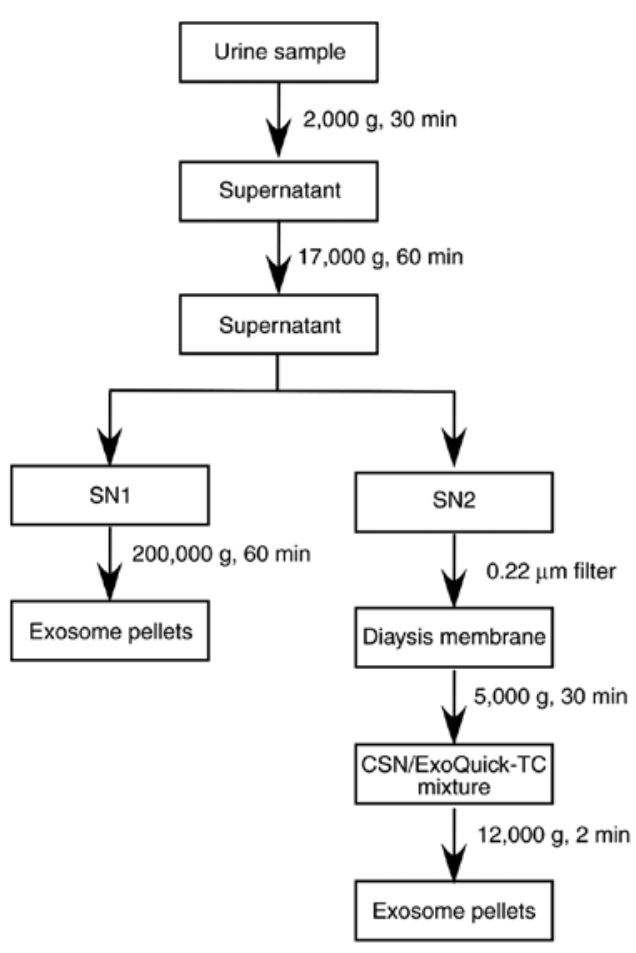

Figure 1. Urinary exosomes isolated by OUF and UC. Workflow to isolate urinary exosomes from urine samples obtained from healthy volunteers. UC, ultracentrifugation; OUF, optimized ultrafiltration; SN, supernatant; CSN, concentrated supernatant.

An unpaired Student's t-test was used to compare the miRNA expression in different groups. $\mathrm{P}<0.05$ indicated that a difference was statistically significant. All results have been verified three times.

\section{Results}

Exosome purity and abundance. UC and OUF were used to isolate exosomes from the same volume of urine. The results demonstrated that pellets (denoted by the black arrow in Fig. 2A) isolated from the OUF group appeared to be larger in comparison with those from the UC group. To ensure that the pellets isolated by OUF and UC were indeed exosomes, they were identified by western blotting, TEM and NTA. The results of western blotting demonstrated the presence of canonical exosome proteins CD63 and Hsp70 in the isolated sediments, which confirmed that OUF and UC successfully isolated exosomes from the urine samples (Fig. 2B).

TEM analysis was used to observe the morphology and size of exosomes. Notably, the TEM images of the OUF group revealed that exosomes displayed a cup-shaped morphology, with relative sizes ranging between 30 and $100 \mathrm{~nm}$ (Fig. 3A and B). However, fewer exosomes were detected in the field of vision in the UC group when compared with the OUF group. The majority of the vesicles isolated from the UC group presented as larger particles that were white, without the classical exosome morphology and with a diameter of 50-200 nm (Fig. 3A). In addition, the TEM images of exosomes isolated in the three different collection periods were compared. The results indicated that the exosomes in the morning group exhibited better morphology in both UC and

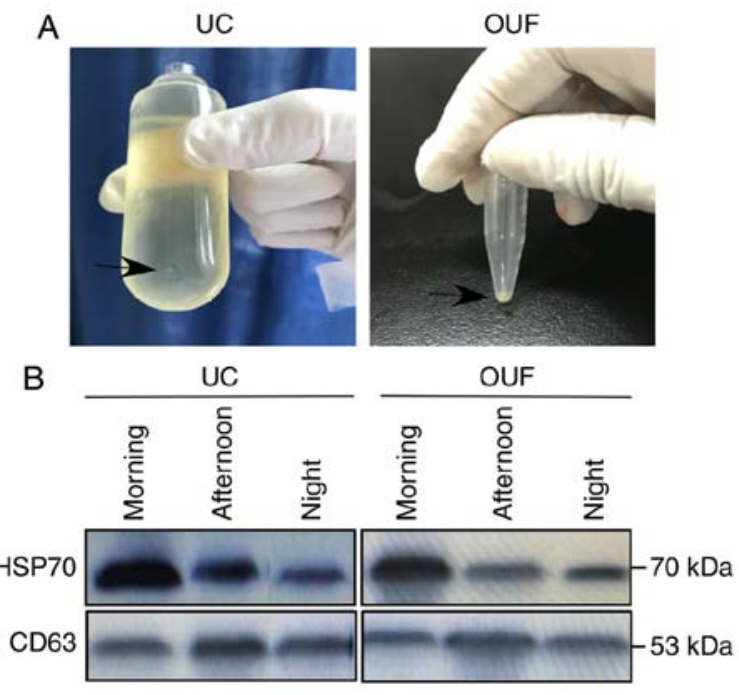

Figure 2. Existence of exosomes in pellets, detected by western blotting (A) Exosomes from urine samples were respectively isolated by UC and OUF. Pellets (black arrow) are translucent and loosely bound to the tube. (B) Western blotting was used to detect the presence of exosomes. Analysis of exosomal protein indicated that CD63 and Hsp70 levels were detectable in the pellets isolated by UC and OUF. UC, ultracentrifugation; OUF, optimized ultrafiltration.

OUF, and it is thus proposed that morning would be the best time for collecting urine samples for exosome isolation.

Next, NTA was used to measure the size distribution of particles consistent with the size range of exosomes. The results revealed that these vesicles ranged in size between 50 and $250 \mathrm{~nm}$. NTA also demonstrated that the exosomes derived from the morning group constituted a higher proportion of the total vesicles as compared with those in the afternoon and evening groups in both UC and OUF, which again proved that morning is the best time for collecting urine samples for the isolation of exosomes (Fig. 4). Furthermore, as seen in Fig. 4, the exosomes (30-100 nm) isolated by OUF constituted a significantly higher proportion of the total vesicles as compared with those obtained from UC in all three time periods (morning, 68.5 vs. 98.5\%; afternoon, 21.7 vs. 91.3\%; evening, 9.8 vs. 95.8\%; Fig. 4). These results clearly suggested the existence of exosomes in urine and the superiority of OUF over UC for isolating these exosomes.

Stability of the biological function and structural integrity of exosomes. To visualize the difference in protein levels between UC and OUF fractions, the same volumes of urine samples isolated by these two protocols were treated under the same conditions. A protein sample of the exosomes of approximately $20 \mu \mathrm{l}$ was loaded onto a colloidal Coomassie-stained gel (Fig. 5A). In the OUF group, there was increased protein expression as compared with that in the UC group under identical culture conditions (Fig. 5A).

Recent studies have demonstrated that exosome-derived miRNAs mediate the communication between a tumor and the surrounding microenvironment, and suggested that they are potential biomarkers of cancer $(26,36)$. In addition, Li et al (27) indicated that the increase of miR-205 was significantly correlated with poor survival outcome in ovarian cancer. Furthermore, miR-7-5p has been found to be 
A

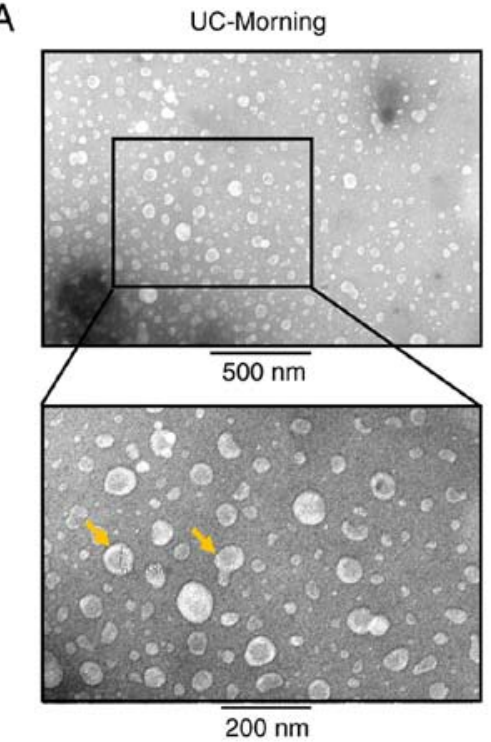

B

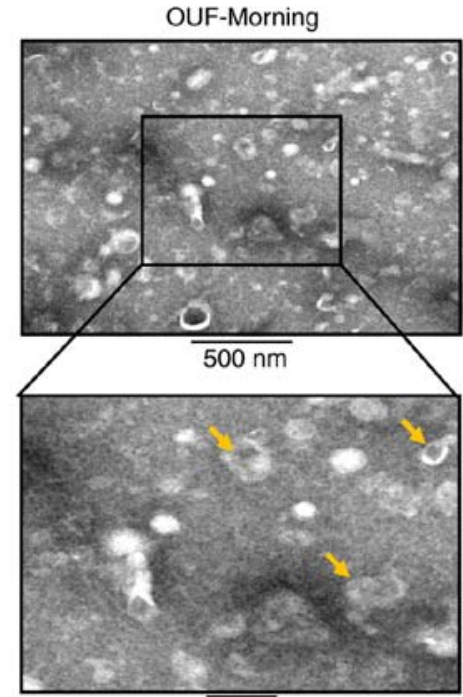

$\overline{200 \mathrm{~nm}}$

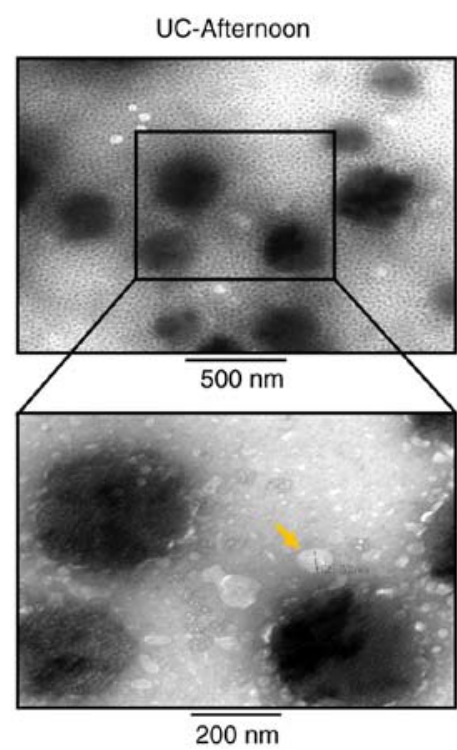

OUF-Afternoon

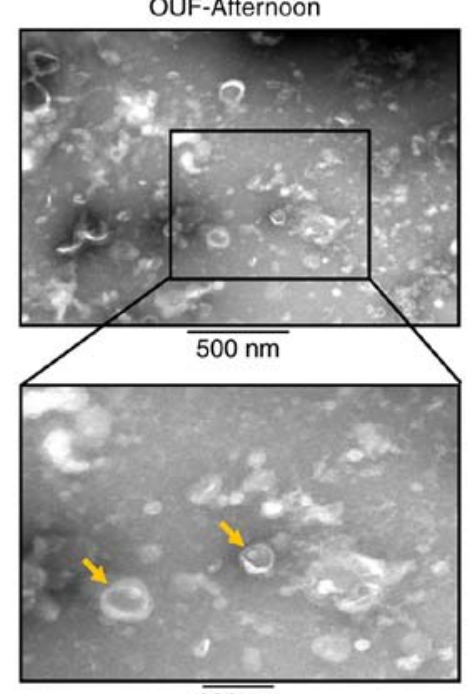

$\overline{200 \mathrm{~nm}}$
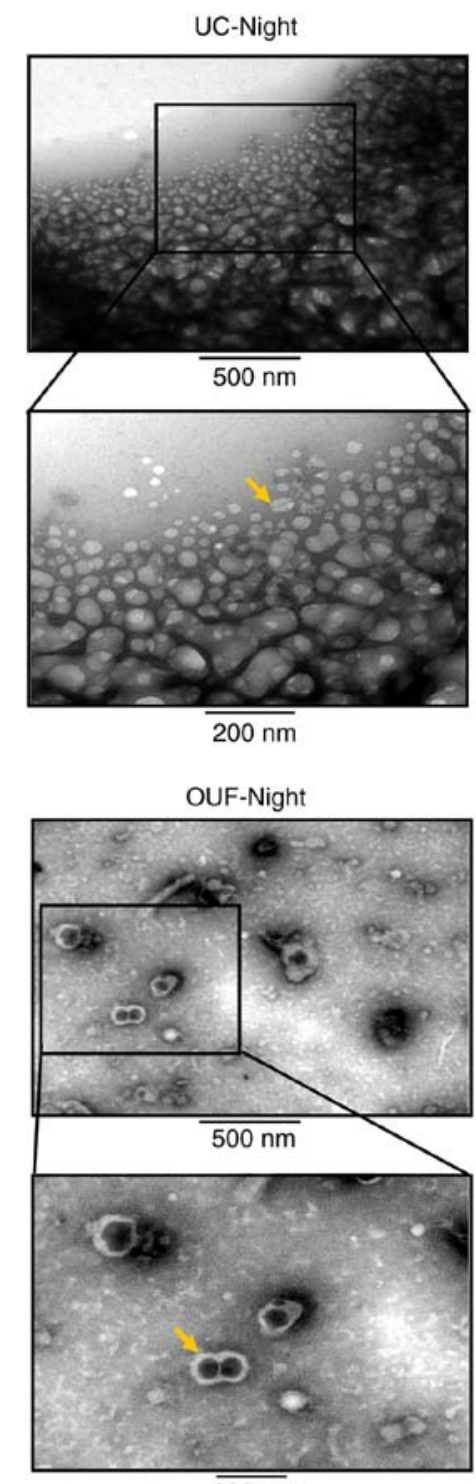

$\overline{200 \mathrm{~nm}}$

Figure 3. Transmission electron microscopy analysis of urinary exosomes isolated using (A) UC and (B) OUF. Upper images show low magnification (x5,000) of exosomes (scale bar, $500 \mathrm{~nm}$ ), while bottom images are at higher magnification (x20,000; scale bar, $200 \mathrm{~nm})$. Yellow arrows indicate the exosomes. UC, ultracentrifugation; OUF, optimized ultrafiltration.

downregulated in breast cancer and may function as a potential prognostic biomarker of breast cancer (37). Therefore, in the present study, exosomal miRNAs were purified, and RT-qPCR was performed to detect the expression of these two miRNAs, hsa-miR-205 and hsa-miR-7-5p. The results revealed that the expression levels of miR-205 and miR-7-5p in urinary exosomes isolated by OUF were significantly higher compared with those isolated by UC at all time points $(\mathrm{P}<0.05$; Fig. $5 \mathrm{~B})$.

\section{Discussion}

In recent decades, numerous studies illustrating the structure and function of exosomes have been conducted. Early studies favored the notion that exosomes may function as cellular garbage bags storing excess material and have no physiological functions (2-4). At present, increasing research suggests that exosomes serve a significant role in cell-to-cell interaction and have great potential as biomarkers for a number of diseases.
However, severe issues associated with the inefficiency of nonstandardized exosome isolation protocols remain unresolved, limiting associated research and applications. UC, the most widely used exosome isolation strategy, has long been considered the gold standard for this purpose $(38,39)$. However, this method has various procedural issues, including a long process, the need for costly ultracentrifuges and continuous UC steps, which inevitably damage the isolated exosomes. As a popular alternative protocol, the synthetic polymer-based precipitation method is relatively simple and produces a large amount of exosomes (40); however, the cost of this method is high, and it usually provides exosomes with low purity and stability. In the present study, we propose a simple and efficient method for addressing these problems.

Urine samples were first centrifuged at 2,000 and $17,000 \times \mathrm{g}$ to remove cellular debris, bacteria, urinary casts and the majority of the THP. The exosomal vesicles can be obtained by direct UC at $200,000 \times \mathrm{g}$ in the UC group, 
A Exosomes $(30-100 \mathrm{~nm}): 68.5 \%$

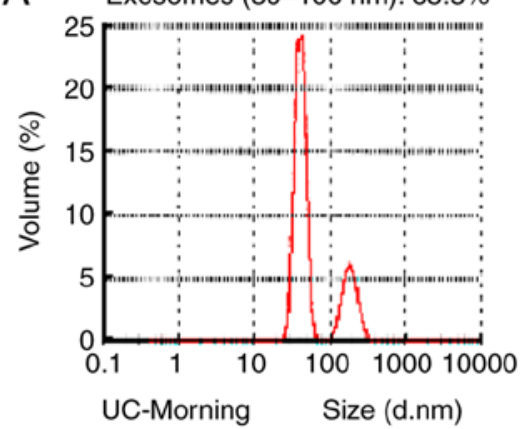

B

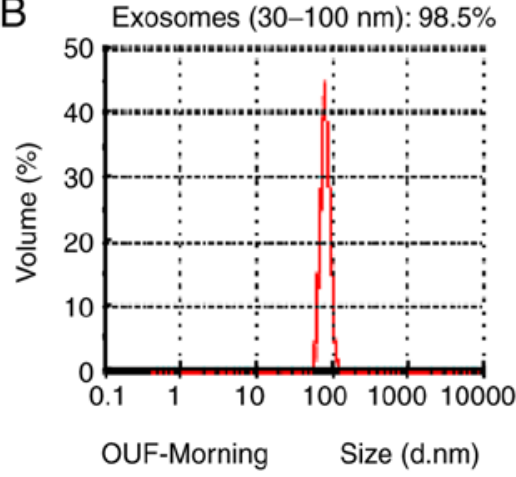

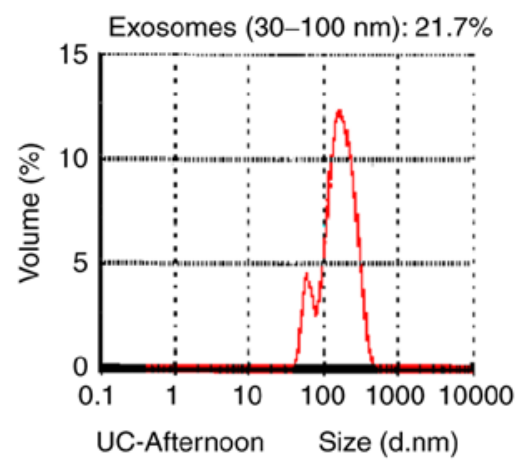

Exosomes (30-100 nm): $91.3 \%$

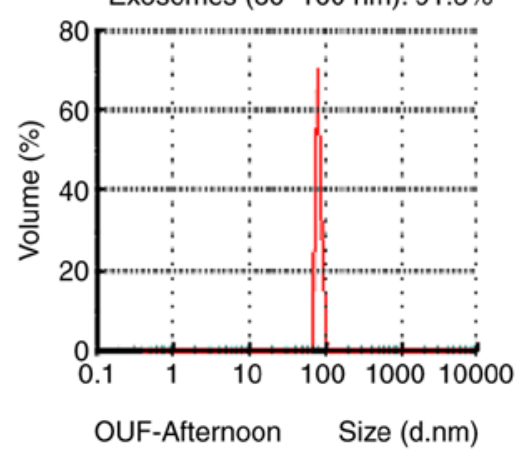

Exosomes $(30-100 \mathrm{~nm}): 9.8 \%$

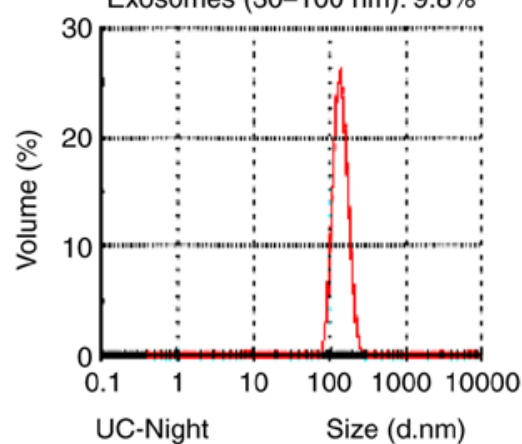

Exosomes (30-100 nm): 95.8\%

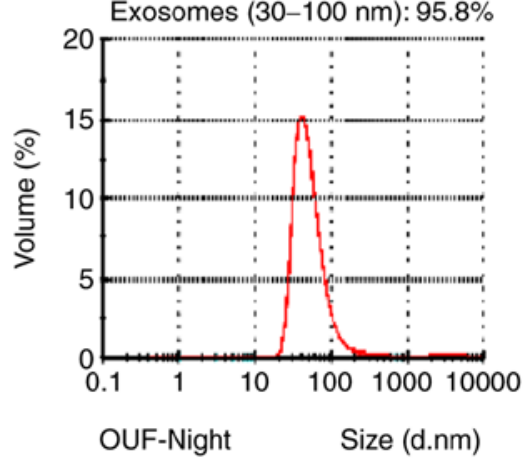

Figure 4. NanoSight nanoparticle tracking analysis conducted to determine the size distribution of urinary exosomes isolated by (A) UC and (B) OUF. UC, ultracentrifugation; OUF, optimized ultrafiltration.
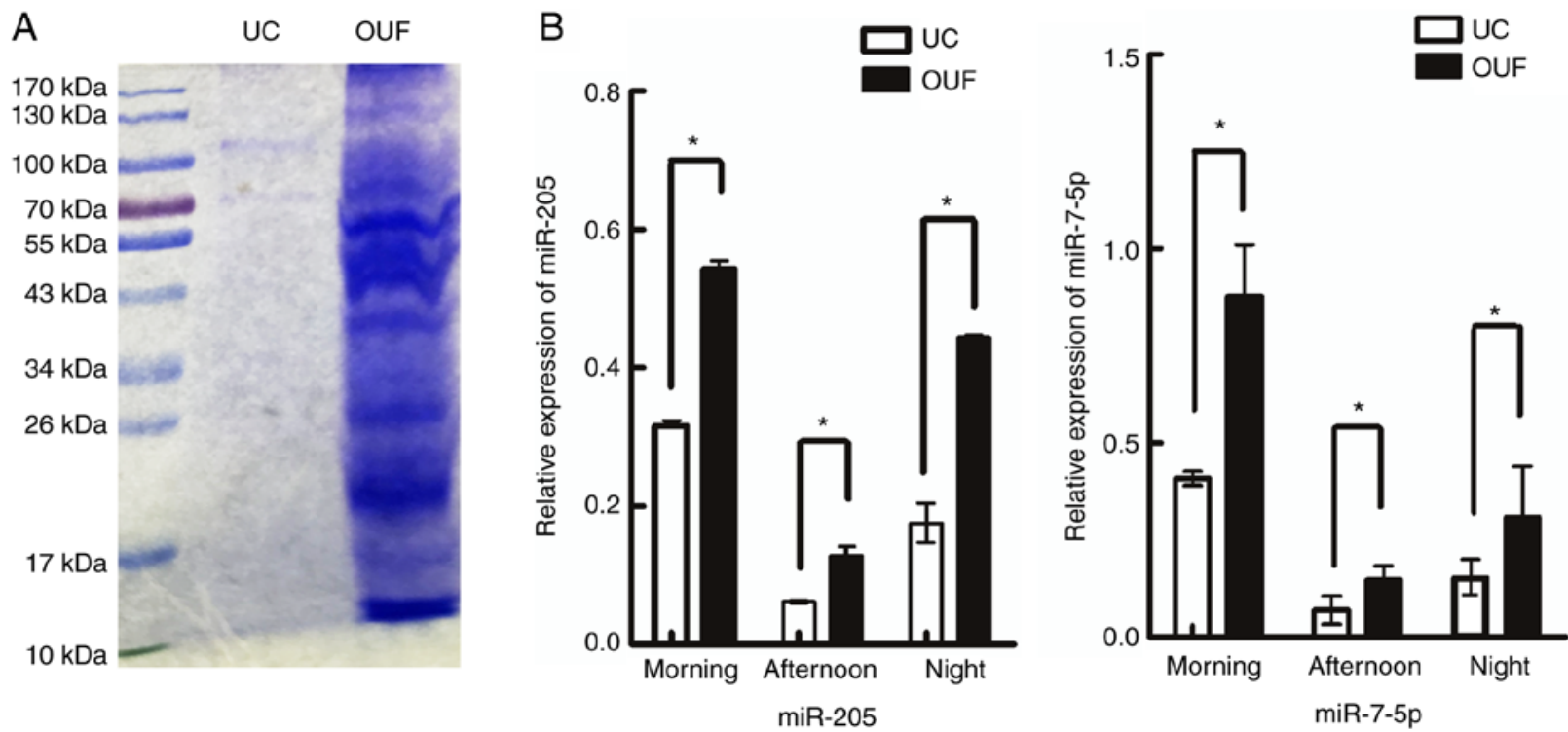

Figure 5. Stability and structure integrity of exosome. (A) SDS-PAGE protein pattern. Colloidal Coomassie-stained gel of exosome fractions isolated by UC and OUF. In total, 20- $\mu 1$ urinary exosomes were loaded per lane in the order of morning, middle and night. Sizes of reference bands are indicated on the left in kilodaltons (kDa). (B) RT-qPCR detection of miR-205 and miR-7-5p levels in urinary exosomes from health donor samples that were collected at three different time and respectively isolated by UC and OUF. Results are expressed as the mean \pm standard deviation ( $\mathrm{n}=3 ; 3$ biological replicates, with 3 technical replicates each). ${ }^{*} \mathrm{P}<0.05$. UC, ultracentrifugation; OUF, optimized ultrafiltration.

however, the intense centrifugal force may destroy the integrity of exosomes. To minimize the damage to exosomes produced by the centrifugal force as much as possible, a filtration step with a $0.22-\mu \mathrm{m}$ filter was used to purify SN2. Next, a dialysis membrane with MWCO of $10,000 \mathrm{kDa}$ was introduced to concentrate the filtered fluids up to $1 / 50$ of the initial volume. This concentration step was aimed to reduce the consumption of ExoQuick-TC and the incubation time of CSN and ExoQuick-TC mixture. Subsequently, ExoQuick-TC was incubated with CSN to gently precipitate the exosomes from the mixture (Fig. 1). The aforementioned improvements included in the OUF method resulted in the production of more exosomes, which were of higher purity and biological stability, as demonstrated through various characterization procedures. 
Table I. Comparative performance of UC and OUF.

\begin{tabular}{|c|c|c|}
\hline Method & $\mathrm{UC}$ & OUF \\
\hline Detectable CD63 and Hsp70 levels & Yes & Yes \\
\hline Pellet size & Small & Large \\
\hline TEM analysis & $\begin{array}{l}\text { Less exosomes, contains numerous } \\
\text { non-exosome particles }\end{array}$ & $\begin{array}{l}\text { More exosomes, contains few } \\
\text { non-exosome particles }\end{array}$ \\
\hline NTA analysis & $\sim 33.33 \%$ exosomes in all vesicles & $\sim 95.2 \%$ exosomes in all vesicles \\
\hline Protein expression pattern & Low protein levels & Increased protein levels \\
\hline Expression of miR-205 and miR-7-5p & Low & High \\
\hline Time consumed & $150 \min$ & $152 \min$ \\
\hline Cost & $\begin{array}{l}\text { Approximately } ¥ 400 \text { (60-ml sample) } \\
\text { (matched tube } x 2=¥ 100 \text {; UC time: } \\
1 \mathrm{~h}=¥ 300 \text { ) }\end{array}$ & $\begin{array}{l}\text { Approximately } ¥ 240(60 \text {-ml sample) } \\
\text { (ExoQuick-TC volume: } 200 \mu 1=¥ 50 \text {; MWCO } \\
\text { tube } \mathrm{x} 2=¥ 180 ; 0.22 \mu \mathrm{m} \text { filter }=¥ 10 \text { ) }\end{array}$ \\
\hline
\end{tabular}

UC, ultracentrifugation; OUF, optimized ultrafiltration; Hsp70, heat shock protein 70; TEM, transmission electron microscope; NTA, nanoparticle tracking analysis; miR, microRNA; $¥$, Chinese Yuan.

The exosome marker proteins CD63 and Hsp70 were detectable in the exosome pellets, indicating that UC and OUF were both able to isolate a considerable amount of exosomes (Fig. 2B). However, according to the results shown in Fig. 2, the exosome pellets harvested from the OUF group were clearly larger in comparison with those from the UC group (Fig. 2B). These results indicated that use of the OUF method extracted a higher number of exosomes. For further analysis of these two methods regarding the purity and quantity of isolated exosomes, these exosomes were observed by TEM and NTA, respectively. The results of TEM revealed that exosomes had a clear cup-shaped morphology with a diameter of 100-130 nm (Fig. 3A and B). Few typical exosome-like vesicles were identified in the UC group, and the majority of these particles were heterogeneous $(50-200 \mathrm{~nm})$, without a classical exosome structure (Fig. 3A). By contrast, TEM images of the OUF group demonstrated a high concentration of exosomes, which were relatively homogeneous with a size of $100-130 \mathrm{~nm}$ (Fig. 3B). These results indicated that the purity of the exosomes isolated by OUF was higher in comparison with that of UC-isolated exosomes. Subsequently, NTA was applied to measure the proportion of vesicles consistent with the size range of exosomes. This revealed that the mean proportion of exosomes in the OUF group (95.2\%) was higher compared with that in the UC group (33.33\%; Fig. 4). This suggested that the abundance of exosomes isolated by OUF was higher as compared with that obtained by UC.

It is well known that exosomes containing specific proteins and miRNA are likely to be carriers of information between cells, and thus the stability of biological functions and structural integrity of exosomes are very important for their further study and clinical application. Therefore, in the current study, the protein and miRNA expression levels in exosomes were measured. The SDS-PAGE protein pattern in the OUF group exhibited increased protein levels compared with those in the UC group (Fig. 5A), while the quantities of miR-205 and miR-7-5p in urinary exosomes isolated by OUF were all significantly higher compared with those in UC $(\mathrm{P}<0.05$; Fig. 5B). These findings indicate that the OUF method may prevent damage to the stability and integrity of exosomes. Finally, the time and cost between UC and OUF in the entire process of isolating exosomes were compared (Table I). The results implied that high-quality exosomes were obtained by OUF in a similar time and lower cost than when UC was performed (Table I).

In conclusion, the present study successfully developed a highly efficient method to isolate urinary exosomes with high purity, production and biological stability. Compared with conventional UC, OUF offers a simple alternative for harvesting high-quality urinary exosomes using a simpler method. Furthermore, the miRNA expression in OUF exosomes was high, which may be suitable for the large-scale extraction of clinical urine samples and subsequent experiments, such as small RNA sequencing and RT-qPCR. It is noteworthy that the OUF approach is more suitable for the isolation of exosomes from samples with a large amount of liquid, such as urine or supernatant of cells, whereas it may not suitable for blood or other specimens with less fluid, which may inevitable have some sample lost in the procedure of isolation. The development of a functional filter that can concentrate and reduce the amount of sample lost is ongoing in our laboratory.

\section{Acknowledgements}

We thank Liwen Bianji of Edanz Group China for language editing the manuscript draft.

\section{Funding}

This work was supported by grants from the National Natural Science Foundation of China (grant no. 81172469) and Technology Program of Changsha City Science Technology Bureau (grant no. K1403050-31). 


\section{Availability of data and materials}

The analyzed and/or datasets generated during the study are available from the corresponding author on reasonable request.

\section{Authors' contributions}

LH conceived and designed the study, performed the experiments, processed the data and wrote the paper. DZ and JW performed the experiments and processed the data. XW contributed to the study design, processed the data, and reviewed and edited the drafts of the paper. All authors provided help during the research, including providing subject design, data acquisition and analysis, article drafting and writing assistance. All authors read and approved the final manuscript.

\section{Ethics approval and consent to participate}

The present study was approved by the Ethics Committee of Central South University (Changsha, China) and conducted in adherence with the Declaration of Helsinki.

\section{Patient consent for publication}

Not applicable.

\section{Competing interests}

The authors declare that there are no conflicts of interest regarding the publication of this manuscript.

\section{References}

1. Trams EG, Lauter CJ, Salem N Jr and Heine U: Exfoliation of membrane ecto-enzymes in the form of micro-vesicles. Biochim Biophys Acta 645: 63-70, 1981.

2. Harding C, Heuser J and Stahl P: Endocytosis and intracellular processing of transferrin and colloidal gold-transferrin in rat reticulocytes: Demonstration of a pathway for receptor shedding. Eur J Cell Biol 35: 256-263, 1984.

3. Johnstone RM, Adam M, Hammond JR, Orr L and Turbide C: Vesicle formation during reticulocyte maturation. Association of plasma membrane activities with released vesicles (exosomes). J Biol Chem 262: 9412-9420, 1987.

4. Mathivanan S, Ji H and Simpson RJ: Exosomes: Extracellular organelles important in intercellular communication. J Proteomics 73: 1907-1920, 2010.

5. Bao L, You B, Shi S, Shan Y, Zhang Q, Yue H, Zhang J, Zhang W, Shi Y, Liu Y, et al: Metastasis-associated miR-23a from nasopharyngeal carcinoma-derived exosomes mediates angiogenesis by repressing a novel target gene TSGA10. Oncogene 37: 2873-2889, 2018.

6. Chen L, Lu FB, Chen DZ, Wu JL, Hu ED, Xu LM, Zheng MH, Li H, Huang Y, Jin XY, et al: BMSCs-derived miR-223-containing exosomes contribute to liver protection in experimental autoimmune hepatitis. Mol Immunol 93: 38-46, 2018.

7. Rajendran L, Bali J, Barr MM, Court FA, Krämer-Albers EM, Picou F, Raposo G, van der Vos KE, van Niel G, Wang $J$ and Breakefield XO: Emerging roles of extracellular vesicles in the nervous system. J Neurosci 34: 15482-15489, 2014.

8. Pathare G, Dhayat N, Mohebbi N, Wagner CA, Cheval L, Neuhaus TJ and Fuster DG: Acute regulated expression of pendrin in human urinary exosomes. Pflugers Arch 470: 427-438, 2018.

9. Bai R, Latifi Z, Kusama K, Nakamura K, Shimada M and Imakawa K: Induction of immune-related gene expression by seminal exosomes in the porcine endometrium. Biochem Biophys Res Commun 495: 1094-1101, 2018.
10. Hu Y, Rao SS, Wang ZX, Cao J, Tan YJ, Luo J, Li HM, Zhang WS, Chen CY and Xie H: Exosomes from human umbilical cord blood accelerate cutaneous wound healing through miR-21-3p-mediated promotion of angiogenesis and fibroblast function. Theranostics 8: 169-184, 2018

11. 11.Asea A, Jean-Pierre C, Kaur P, Rao P, Linhares IM, Skupski D and Witkin SS: Heat shock protein-containing exosomes in mid-trimester amniotic fluids. J Reprod Immunol 79: 12-17, 2008.

12. Zomer A, Maynard C, Verweij FJ, Kamermans A, Schäfer R, Beerling E, Schiffelers RM, de Wit E, Berenguer J, Ellenbroek SI, et al: In Vivo imaging reveals extracellular vesicle-mediated phenocopying of metastatic behavior. Cell 161: 1046-1057, 2015

13. Zhang H, Wang Y, Bai M, Wang J, Zhu K, Liu R, Ge S, Li J, Ning T, Deng T, et al: Exosomes serve as nanoparticles to suppress tumor growth and angiogenesis in gastric cancer by delivering hepatocyte growth factor siRNA. Cancer Sci 109: 629-641, 2018.

14. Ferguson Bennit HR, Gonda A, McMullen JRW, Kabagwira J and Wall NR: Peripheral blood cell interactions of cancer-derived exosomes affect immune function. Cancer Microenviron: Mar 30, 2018 (Epub ahead of print).

15. Sung BH, Ketova T, Hoshino D, Zijlstra A and Weaver AM: Directional cell movement through tissues is controlled by exosome secretion. Nat Commun 6: 7164, 2015.

16. Costa-Silva B, Aiello NM, Ocean AJ, Singh S, Zhang H, Thakur BK, Becker A, Hoshino A, Mark MT, Molina H, et al: Pancreatic cancer exosomes initiate pre-metastatic niche formation in the liver. Nat Cell Biol 17: 816-826, 2015.

17. Armstrong EA, Beal EW, Chakedis J, Paredes AZ, Moris D, Pawlik TM, Schmidt CR and Dillhoff ME: Exosomes in pancreatic cancer: From early detection to treatment. J Gastrointest Surg 22: 737-750, 2018.

18. Cui S, Cheng Z, Qin W and Jiang L: Exosomes as a liquid biopsy for lung cancer. Lung Cancer 116: 46-54, 2018.

19. Murakami T, Oakes M, Ogura M, Tovar V, Yamamoto C and Mitsuhashi M: Development of glomerulus-, tubule-, and collecting duct-specific mRNA assay in human urinary exosomes and microvesicles. PLoS One 9: e109074, 2014.

20. Fernández-Llama P, Khositseth S, Gonzales PA, Star RA, Pisitkun T and Knepper MA: Tamm-Horsfall protein and urinary exosome isolation. Kidney Int 77: 736-742, 2010.

21. Pathare G, Dhayat NA, Mohebbi N, Wagner CA, Bobulescu IA, Moe OW and Fuster DG: Changes in V-ATPase subunits of human urinary exosomes reflect the renal response to acute acid/alkali loading and the defects in distal renal tubular acidosis. Kidney Int 93: 871-880, 2018.

22. Kesimer M and Gupta R: Physical characterization and profiling of airway epithelial derived exosomes using light scattering. Methods 87: 59-63, 2015.

23. Alvarez ML, Khosroheidari M, Kanchi Ravi R and DiStefano JK: Comparison of protein, microRNA, and mRNA yields using different methods of urinary exosome isolation for the discovery of kidney disease biomarkers. Kidney Int 82: 1024-1032, 2012.

24. Zhao W, Zheng XL and Zhao SP: Exosome and its roles in cardiovascular diseases. Heart Fail Rev 20: 337-348, 2015.

25. Tran TH, Mattheolabakis G, Aldawsari H and Amiji M: Exosomes as nanocarriers for immunotherapy of cancer and inflammatory diseases. Clin Immunol 160: 46-58, 2015.

26. Zhou J, Gong G, Tan H, Dai F, Zhu X, Chen Y, Wang J, Liu Y, Chen $\mathrm{P}, \mathrm{Wu} \mathrm{X}$ and Wen J: Urinary microRNA-30a-5p is a potential biomarker for ovarian serous adenocarcinoma. Oncol Rep 33: 2915-2923, 2015.

27. Li J, Hu K, Gong G, Zhu D, Wang Y, Liu H and Wu X: Upregulation of MiR-205 transcriptionally suppresses SMAD4 and PTEN and contributes to human ovarian cancer progression. Sci Rep 7: 41330, 2017.

28. Li J, Li L, Li Z, Gong G, Chen P, Liu H, Wang J, Liu Y and Wu X: The role of miR-205 in the VEGF-mediated promotion of human ovarian cancer cell invasion. Gynecol Oncol 137: 125-133, 2015.

29. Musante L, Tataruch D, Gu D, Benito-Martin A, Calzaferri G, Aherne $\mathrm{S}$ and Holthofer $\mathrm{H}$ : A simplified method to recover urinary vesicles for clinical applications, and sample banking. Sci Rep 4: 7532, 2014.

30. Colombo M, Moita C, van Niel G, Kowal J, Vigneron J, Benaroch P, Manel N, Moita LF, Théry C and Raposo G: Analysis of ESCRT functions in exosome biogenesis, composition and secretion highlights the heterogeneity of extracellular vesicles. J Cell Sci 126: 5553-5565, 2013 . 
31. Lv LL, Cao Y, Liu D, Xu M, Liu H, Tang RN, Ma KL and Liu BC: Isolation and quantification of microRNAs from urinary exosomes/microvesicles for biomarker discovery. Int J Biol Sci 9: 1021-1031, 2013.

32. Bradford MM: A rapid and sensitive method for the quantitation of microgram quantities of protein utilizing the principle of protein-dye binding. Anal Biochem 72: 248-254, 1976.

33. Tkach M, Kowal J,Zucchetti AE, Enserink L, Jouve M, Lankar D, Saitakis M, Martin-Jaular L and Théry C: Qualitative differences in T-cell activation by dendritic cell-derived extracellular vesicle subtypes. EMBO J 36: 3012-3028, 2017.

34. Candiano G, Bruschi M, Musante L, Santucci L, Ghiggeri GM, Carnemolla B, Orecchia P, Zardi L and Righetti PG: Blue silver: A very sensitive colloidal Coomassie G-250 staining for proteome analysis. Electrophoresis 25: 1327-1333, 2004.

35. Livak KJ and Schmittgen TD: Analysis of relative gene expression data using real-time quantitative PCR and the 2(-Delta Delta C(T)) method. Methods 25: 402-408, 2001

36. Casadei L, Calore F, Creighton CJ, Guescini M, Batte K, Iwenofu OH, Zewdu A, Braggio DA, Bill KL, Fadda $\mathrm{P}$, et al: Exosome-derived miR-25-3p and miR-92a-3p stimulate liposarcoma progression. Cancer Res 77: 3846-3856, 2017.
37. Block I, Burton M, Sørensen KP, Andersen L, Larsen MJ, Bak M, Cold S, Thomassen M, Tan Q and Kruse TA: Association of miR-548c-5p, miR-7-5p, miR-210-3p, miR-128-3p with recurrence in systemically untreated breast cancer. Oncotarget 9 : 9030-9042, 2018.

38. Lai RC, Yeo RW, Tan KH and Lim SK: Mesenchymal stem cell exosome ameliorates reperfusion injury through proteomic complementation. Regen Med 8: 197-209, 2013.

39. Gould SJ and Raposo G: As we wait: Coping with an imperfect nomenclature for extracellular vesicles. J Extracell Vesicles 2, 2013.

40. Xu R, Greening DW, Zhu HJ, Takahashi N and Simpson RJ: Extracellular vesicle isolation and characterization: Toward clinical application. J Clin Invest 126: 1152-1162, 2016.

(7)(9) This work is licensed under a Creative Common Attribution-NonCommercial-NoDerivatives 4.0 International (CC BY-NC-ND 4.0) License. 